\title{
Modern techniques of trend analysis and interpolation
}

\author{
L. TORELLI $(*)$
}

Received on August 20th, 1975

StmmaRr. -- This article contains a schematic exposition of the theoretical framework on which recent techniques of trend analysis and interpolation rest.

It is shown that such techniques consist in the joint application of Analysis of the Variance and of Mrultivariate Distribution Analysis. The theory of Universal Kriging by G. Watheron is also discussed and reduced to the above theories.

Riassunto. - Il presente articolo espone in forma schematica i risultati teorici su cui si basano le tecniche più recenti di interpolazione e analisi di tendenza.

Viene dimostrato che tali tecniche risultano dall'applicazione congiunta di Analisi della Varianza e di Analisi Multivariata. Si discute inoltre la teoria del "Irigeage Universel" di G. Matheron, riducendola alle teorie già men. zionate.

\section{1. - Introduction}

One deals with a function $y(x)$ defined in a region of a real space. The value of the function is known at a few points, $x_{i}$, of the region. One writes $y_{i}=y\left(x_{i}\right)$. Interpolation means prediction of the value of the function at points of the region where the value of the function is unknown. Trend is a simplified function capable to describe the "general behavior" of the original function. These concepts are better" defined when the theoretical approach to the problem is described.

(*) IDROTECNECO, Rome. 
One consirlers the function in question as a realization of a stochastic function $T(x)$ with the following structure:

$$
\Gamma(x)=f(x)+\varepsilon(x) ; \quad E \varepsilon(x) \stackrel{r}{=} 0
$$

where $f(x)$ is a deterministic function describing the expectation $E Y(x)$ of $Y(x)$ and $\varepsilon(x)$ is a stochastic resiclual with expectation zero.

$f(x)$ is what is usually called trend of $y(x)$ and the problem of trend analysis is to find an estimate as good as possible of $f(x)$. It will be shown that it is possible to find an unbiased estimator which has minimum variance in the class of the linear estimators of $f(x)$.

As to interpolation, one wants to find an optimal predictor of $Y(x)$, i.e. a prerlictor $\mathcal{I}(x)$ such that:

$$
\begin{aligned}
& E\left(Y^{\prime}(x)-Y(x)\right)=0 \\
& E(Y(x)-I(x))^{2} \text { is minimum. }
\end{aligned}
$$

Section 2 gives a schematic account of the theory which can be used to attrin the above objectives. It is articulater in three parts, one about trend analysis, one about interpolation when $f(x) \stackrel{x}{=} 0$ and the last about interpolation in general. The theory used is the Analysis of the Variance (Scheffe, 1959) (8) and Multivariate Distribution Analysis (Anderson, 1958) ().

Section 3 discusses a theory elaborater in the late sixties by Matheron and co-workers called Universal Kriging (Matheron, 1969 (5), $^{(5)}$ Matheron, $1971\left({ }^{6}\right)$, Huijbregts and Matheron, $1970\left({ }^{4}\right)$ and reduces it to the theory presented in section 2. Conclusions are drawn in section 4 .

\section{2. - SCHEME OF THE THEORY}

\section{1. - Trend Analysis}

Consider equation [1]. If $f(x)$ is formulated as a linear function, with known coefficients function of $x$, of a number of parameters to be estimated, one can make use of the Analysis of the Variance to solve the problem of estimating $f(x)$.

In facts one constructs a linear morlel, which in matrix notation is expressed as follows:

$$
y=X^{\prime \prime} \beta+\varepsilon, \quad E \varepsilon=0, \quad \Sigma_{y}=\Sigma_{\varepsilon}=B
$$


where $y$ is the vector of the observations, $\beta$ the vector of the parameters to be estinated, $\varepsilon$ the vector of the stochastic residuals and $\mathrm{I}^{\prime}$ a full rank matrix whose ieth row is composed by the coefficients of the linear expression of $f\left(x_{i}\right)$ in $\beta .^{\prime}$ means transposed and $\Sigma_{\varepsilon} \Sigma_{y}$ are the covariance matrices of $\varepsilon$ and $y$ respectively and are assumed known.

Given model 2, the following results follow (Schefle, 1959) ${ }^{8}$ ).

1) $\hat{\beta}=\left(X B^{-1} \mathrm{I}^{\prime}\right)^{-1} \mathrm{X} B^{-1} y$ is an unbiased estimator of $\beta$ which has minimum variance in the class of the linear estimators of $\beta$. It should be noted that $\dot{\beta}$ is diflerent from the usual least square estimator of $\beta$,

$$
\hat{\beta}=\left(I I^{\prime}\right)^{-1} \mathbf{I} y
$$

$\hat{\beta}$ is unbiaser but has not minimum variance in the class of the linear estimator's of $\beta$.

2) (Gauss Markov Theorem) Given a vector $a$ if there exists a vector $c$ such that $a=c X^{\prime}, a \beta$ is the unique unbiased estimator of $a \beta$ which has minimum variance in the class of the linear estimators of $a \beta$.

Since $f(x)$ is linear in $\beta$, we get

$$
f(x)=a(x) \beta
$$

where $a(x)$ satisfies the condition of the Gauss Markor Theorem because $\mathrm{I}^{\prime}$ is full rank. Consequently, through the Analysis of the Variance, one obtains an estimator $f(x)=a(x) \dot{\beta}$ of $f(x)$ which is optimal in the class of the linear estimators of $f(x)$. Moreover, the theory gives the matrix of the covariance of $\bar{\beta}$

$$
\Sigma_{\beta}=\left(\mathrm{IB}^{-1} \mathrm{I}^{\prime}\right)^{-1}
$$

so that the variance of the estimator $a(x) \hat{\beta}$ of $f(x)$ is $a(x)\left(X B^{-1} X^{\prime}\right)^{-1} a(x)^{\prime}$.

2.2. - Interpolation when $f(x) \stackrel{x}{=} 0$

To solve this problem one uses Multivariate Distribution Analysis.

I et us consider the random normal vector $Y^{*}=\left(Y, Y_{1}, Y_{2}, \ldots, Y_{n}\right)^{\prime}$ where $Y=Y(x), x$ being the point of interpolation and $Y_{i}=Y\left(x_{i}\right)$, such that $E Y^{*}=0 . \Sigma_{u^{*}}=\Lambda$ is assumed to be known. 
.1 may be partioned as follows

$$
A=\left|\begin{array}{cc}
c & D^{\prime} \\
D & B
\end{array}\right|
$$

where $B=\sum_{y}$ of the precerling section, $c=\operatorname{var} Y, D=\left(\operatorname{cov}\left(Y, Y_{1}\right)\right.$, $\left.\operatorname{cov}\left(Y, Y_{2}\right), \ldots, \operatorname{cov}\left(Y, Y_{n}\right)\right)^{\prime}$.

One has the following result (Anclerson, 1958) $\left({ }^{2}\right)$.

Given $Y_{i}=y_{i}, i-1,2, \ldots, n, Y$ is normally distributer with mean $S^{\prime}\left(Y / Y_{l}=y_{i} ; i=1,2, \ldots, n\right)=D^{\prime} B^{-1} y$

and variance

$$
\left.\operatorname{var}\left(Y / Y_{i}=u_{i}, i=1,2, \ldots, n\right)=c-D^{\prime} B^{-1} J\right)
$$

$J_{M}(x)-E\left(Y, Y_{i}=y_{i}, i=1,2 \ldots, n\right)$ is obviously an optimal predictor in the sense of section 1 . Subseript $M$ refers to Multivariate. In this methor the assumption of the normality of $I *$ is marle. Such assumption can be considered safe and it is based on Contral Limit Theorem consirlerations.

\section{3. - Interpolation in general}

It is rommon sense to use the following procedure:

one gots an optimal estimate $f(x)$ of $f(x)$ using the theory of section 2.1. Then one applies the methor describer in section 2.2 to the ranfom function $Z(x)=Y(x)-f(x)$. The final interpolator is theil) $f(x)+\hat{Z}_{M}(x)$.

\section{3. - ENentrat Komging}

This theory of interpolation and trend analysis has been developed by Matheron and co-workers in the late sixties. A great mass of literature on the theory has been produced by this school. Only the essential references are given here. In the present riscussion of Universil Kriging, constant reference is male to the article by Huijuregts and Matheron, in which this theory is concisely and clearly exposed. 
The assumption of Huijbregts and Matheron is that one deals with a realization of a stochastic function $Z(x)$ for which the moments of 1 st and 2 nd order are defined:

$$
\begin{aligned}
& E Z(x)=m(x) \\
& E Z(x) Z(y)=C(x, y)+m(x) m(y)
\end{aligned}
$$

where $C(x, y)=\operatorname{cov}(Z(x), Z(y))$.

Moreover

$$
m(x)=\bar{\Sigma}_{1} a_{l} f^{l}(x)(x \varepsilon V)
$$

where $V$ is some neighborhood of the points of observation.

In other words, $m(x)$ is a linear function with known coefficents function of $x$, viz $f^{l}(x)$, of a number of parameters $a_{l}$ to be estimated.

Apparently the structure described by (I,H-M) and $(1, \mathrm{H}-\mathrm{MI})$ is coincident with the linear model of equation [2].

As far as trend analysis is concerned, the proclaimed goal of Huijbregts and Matheron $\left(^{4}\right)$ is to find a linear estimator of $m(x)$ unbiased and with minimum variance. Now, given the fact that the probability structure considered by Huijbregts and Matheron $\left(^{(}\right)$is a linear model and given the unicity property of the estimator of the Gauss Markov theorem, it follows that the Huijbregts and Matheron ( ${ }^{4}$ ) solution is coincident with the standard solution of Analysis of the rariance given in section 2.1.

As to the problem of interpolation in absence of trend, the predictor proposed by Huijbregts and Matheron is

$$
Z_{k}=\Sigma_{\alpha} \lambda_{k}^{\alpha} Z_{\alpha}, \quad \alpha=1,2, \ldots, n
$$

with coefficients $\lambda_{k}^{\alpha}$ verifying the following system

$$
\Sigma_{\beta} \lambda_{k}^{\beta} \sigma_{\alpha \beta}=C\left(\varkappa_{\alpha}, \varkappa_{0}\right)
$$

where $Z_{\alpha}=Z\left(X_{\alpha}\right)$ are the observations, $k$ refers to the point of interpolation, i.e. $x_{0}, \sigma_{\alpha \beta}=C\left(x_{\alpha}, x_{\beta}\right)$ and $\alpha$ runs, like $\beta$, on the number of observations $n$.

If we set $\lambda_{k}=\left(\lambda_{k^{1}}, \lambda_{k^{2}}, \ldots, \lambda_{k^{n}}\right)$, and write, according to the notation of section 2, $y=\left(Z_{1}, Z_{0}, \ldots, Z_{n}\right)$ and $D=\left(C\left(x_{1}, x_{0}\right)\right.$, 
$\left.C\left(x_{2}, x_{0}\right), \ldots, C\left(x_{n}, x_{0}\right)\right)$ and we let $B$ be the covariance matrix of $y$, we an write Huijbregts and Matheron's expressions $\left({ }^{4}\right)$ in matrix notation:

$$
Z_{k}=\lambda_{k}, \quad \lambda_{k} B=D^{\prime}
$$

from which

$$
Z_{k}=D^{\prime} B^{-1} y
$$

coincislent with the solution of section 2.2 .

The IIujbregts and Matheron's solution to the problem of interpolation in general is given by:

$$
Z^{*}=M^{*}\left(x_{0}\right)+\sum_{\alpha} \lambda_{k}^{\alpha}\left(Z_{\alpha}-M^{*}\left(x_{\alpha}\right)\right)
$$

where $I^{*}\left(x_{0}\right)$ is the optimal trend estimator and $\sum_{u} \hat{n}_{k}^{\alpha}\left(Z_{u}-I^{*}\left(x_{u}\right)\right)$ is the solution discussed above for the stochastic function with no trend, $Z(x)-M^{*}(x)$. This is again coincirlent with the solution of section 2.3 .

However, the article of Inijbregts and Matheron (") ofler's a few interesting marginal results, since it shows that $Z_{k}$ is an optimal linear predictor independently of the normality of $Z(x)$ and that the greneral interpolator $(5, \mathrm{H}-\mathrm{MI})$ which is the sum of the optimal linear estimator of the trend and of the optimal linear predictor of the resirluals is itself an optimal linear predictor. Moreover, it provides the overall variance of preslictor $(5, \mathrm{~J}, \mathrm{M})$.

\section{4. - Conclusiox}

This article is meant to be a schematic exposition of the theoretical framework on which recent techniques of trend malysis and interpolation rest. It is shown that such techniques consists in the joint application of the Analysis of the Variance and of Multivariate Distribution Analysis. It is also shown that the bulk of Universal Kriging is reducible to these long established and well known theories. In this we think to have given a contribution to the unification and clarification of the developments in the fiels.

In the paper, the basic probability structure, i.e. the linear morlel, is assumer as given. The construction of a suitable model, which 
implies the determination of the form of the linear expansion of $f(x)$ and of the covariance structure of the resiluals is not discussed, being beyond the scope of the present article.

In any case, this is a rather intricate matter, in which the Analysis of the Variance is again of great help. For an example of inquiry on the covariance structure of a stochastic function defined in a unidimensional discrete space, the reader is referred to Torelli (10) and Torelli and Chow (9).

\section{REFERENCES}

(1) Agterberg F. P., 1974. - "Geomathematies", Elsevier, Amsterdam.

(2) ANDERson T. W., 1958. - An introduction to Multivariate Statistical Analysis. Wiley, New York.

(3) Delfiner P. and J. P. Dethosme, 1973. - Optimum interpolation by Kriging, in Display and Inalysis of Spatial Data. Wiley, New York.

(4) Hetroregts C. and G. Matmerox, 1970. - Universal Friging: an Optimal Method for Estimating and Contouring in Trend Surface Analysis. CIMII, IX Intern. Symp., Montreal.

(5) Matheion G., 1969. - Le Krigeage Universel. "Cahiers du Centre de Iorphologie Mathematiques", 1, Fontainebleau.

(6) Matnezon G., 1971. - The Theory of Regionalized Variables and its Applications. "Cahiers du Centre de Morphologie Mathematique", 5, Fontaincbleau.

(") Otea R. A., 1974. - Optimal Contour Mapping Using Cniversal Kriging. "J.J.R.".

(8) SCmbré II., 1959. - The Analysis of the Variance. Wiley, New York.

(9) Toreu.r 1. and V. T. Cnow, 1972. - Tests of Stationarity of IIydrologic Time series. "Int. Symp. on Uncertainties in IIydrologic and Water Resource Systems", Tueson, December.

(10) Toremin I., 1973. - The Analysis of Monthly Hydrologic Time series. Ph. D. Thesis, Eniversity of Illinois. 